\title{
EXTENSIVE NECROTIZING FASCIITIS OF TRUNK FOLLOWING APPENDICECTOMY
}

\author{
Siddharth Mishra1, Iqbal Ali2 , Gurjit Singh³, Simran Kaur Bhatia ${ }^{4}$
}

\section{HOW TO CITE THIS ARTICLE:}

Siddharth Mishra, Iqbal Ali, Gurjit Singh, Simran Kaur Bhatia. "Extensive Necrotizing Fasciitis of Trunk Following Appendicectomy". Journal of Evolution of Medical and Dental Sciences 2015; Vol. 4, Issue 26, March 30; Page: 4561-4564, DOI: $10.14260 /$ jemds/2015/662

ABSTRACT: Acute appendicitis is usually diagnosed and managed easily with a low mortality and morbidity. However, in $20.74 \%$ patients, acute appendicitis may occasionally become extraordinarily complicated and life threatening with significant morbidity and mortality.[1] We report a case of 44 year old male, a known case of Diabetes Mellitus since 4 years (on irregular treatment) who was brought to our hospital with severe pain in right lower quadrant of abdomen since 4 days. On examination, patient had tachycardia, tenderness and guarding in the right iliac fossa. Laboratory results revealed leucocytosis and raised blood glucose levels. Measures for glycemic control were initiated. At exploratory laparotomy, an inflamed and retrocaecal appendix ruptured at the base with small local abscess was found. Abscess was drained and appendectomy done. Initially patient did well but at the end of second post-operative week, the patient started having pain and swelling over posterior chest wall, flank, sacral and occipital regions suggestive of inflammation. CT abdomen and pelvis showed extensive necrotizing fasciitis of trunk, occiput and sacrum. Repeated multiple fasciotomies were performed over the back. The patient finally recovered fully at the end of $8^{\text {th }}$ postoperative week.

INTRODUCTION: Acute Appendicitis is a disease which is commonly encountered in daily clinical practice. It is one of the significant causes of severe acute abdominal pain worldwide. American surgeon Charles McBurney has established the definite role of early surgical intervention in the management of acute appendicitis.[2] It may sometimes become life threatening. In case the diagnosis and treatment are delayed it gives rise to complications such as peritonitis, perforation and abscess formation. We present an unusual case of retroperitoneal perforated appendicitis resulting in extensive necrotizing fasciitis over back, right flank and nape of the neck, post operatively resulting in prolonged hospital stay.

CASE REPORT: A 44 year old man reported to our hospital with complains of severe pain in right lower quadrant of the abdomen since 4 days. He was a known case of Diabetes Mellitus since 4 years but on irregular treatment. Physical examination revealed an ill-looking man with the body temperature of $38^{\circ} \mathrm{C}$ and tachycardia (pulse rate-100 beats $/ \mathrm{min}$ ).

Tenderness and guarding was present over right iliac fossa. There was leucocytosis with a WBC count of 12,000 cells $/ \mathrm{mm}^{3}$, random blood sugar level was raised $(535 \mathrm{mg} / \mathrm{dL}$ ) and urinary ketone bodies were present (4+). Ultrasound was suggestive of acute appendicitis with no collection in Right Iliac Fossa.

At emergency exploratory laparotomy, appendix was found inflamed and perforated at the base with small collection in the right iliac fossa. Appendectomy was performed. Histopathological examination confirmed Acute Appendicitis. Antibiotics were commenced and glycemic control was achieved. 
The patient had uneventful post-operative course till the end of second post-operative week when he complained of swelling and pain over the right side of posterior chest wall and right flank which subsequently spread to whole sacral and occipital regions. On examination, patient had tachycardia (pulse rate-120 beats/min) although he was afebrile. There was evidence of cellulitis over posterior chest wall, right flank, sacral and occipital regions but no crepitus felt. Ultrasound scan also revealed features of cellulitis while CT scan revealed evidence of extensive necrotizing fasciitis over the trunk. (Fig. 1.)

Multiple incisions and drainage with fasciotomy were performed frank pus was drained (Fig. 2 \& 3). The incisions were left open. The bacterial culture revealed Klebsiella pneumonia which was sensitive to Ciprofloxacin, Chloramphenicol, Gentamicin, Amikacin, Imipenem. He was started on Chloramphenicol 500mg i/v QID, Ciprofloxacin 500mg i/v BID which was accompanied with daily dressing of the wounds. Repeated debridement (Seven times) was required over a period next 7 weeks and by the end of $8^{\text {th }}$ week, patient recovered fully.

DISCUSSION: Intestinal perforation due to appendicitis, diverticulitis, or a carcinoma can cause retroperitoneal and psoas abscesses, which may extend down along the inguinal ligament, or through the femoral canal, sciatic foramen, and obturator foramen, resulting in emphysema and abscesses in the thigh and leg.[3][4][5][6][7] Knowing that the anatomical position of the appendix is variable and $65 \%$ of the appendix have been reported to be retrocecal, ${ }^{[8]}$ a perforation at this location is fraught with danger of retroperitoneal abscess.

In our case, the occurrence of the necrotizing fasciitis of the trunk is very rare. The possible mechanism of the spread of infection is from the retrocaecal region to Petit's triangle to trunk. Post operatively, though there were no abdominal findings (such as guarding and rigidity), the occurrence of signs of inflammation of the trunk region is very unusual.

In case report by Mukoyama et al, an elderly patient with perforated acute appendicitis reported with severe necrotizing fasciitis of the right thorax to right lower abdomen and lateral aspect of the right ilium. ${ }^{[9]}$ They could search only 10 more similar cases from the literature. However, amongst all these none developed necrotizing fasciitis following appendectomy.

In addition to the physical signs of diagnosis, CT scan is used as a diagnostic tool. Contrast enhanced CT scan of the abdomen imaging study gives highest accuracy and efficiency. CT scan of the abdomen not only helps in the establishment of the diagnosis, but also extent of involvement.

CONCLUSION: Extensive Necrotizing Fasciitis of trunk following Appendicectomy is extremely rare. Surgeons should keep this its rare complication in mind which increases morbidity and may result in fatality if not treated aggressively. Incision and drainage with fasciotomy often at multiple sittings with appropriate antibiotics form the mainstay of the management. Emphasis should be laid on early diagnosis and aggressive management.

\section{REFERENCES:}

1. Ahmad T, Ali Z, Ali A, Anjum S. Perforated appendix: contributing factors. JUMDC 2010; 1(2): 11-6.

2. Mc Burney C. Experience with early operation interference in cases of the vermiform appendix. NY State Med 3 1889; 50: 676. 


\section{CASE REPORT}

3. Haiart DC, Stevenson P, Hartley RC. Leg pain: an uncommon presentation of perforated diverticular disease. J R Coll Surg Edinb 1989; 34: 17-20.

4. Kobayashi H, Sakurai Y, Shoji M, Nakamura Y, Suganuma M, Imazu H, et al. Psoas abscess and cellulitis of the right gluteal region resulting from carcinoma of the cecum. J Gastroenterol 2001; 36: 623-8.

5. Nicell P, Tabrisky J, Lindstrom R, Peter M. Thigh emphysema and hip pain secondary to gastrointestinal perforation. Surgery 1975; 78: 555-9.

6. Turner G, Daniell SJ. Lumbar abscess resulting from appendicitis. J R Soc Med 1984; 77: 884-7.

7. Van den Wildenberg FA. The retrocaecal appendix: a snake in the grass. Neth J Surg 1982; 34: 133-5.

8. El-Masry NS, Theodorou NA. Retroperitoneal perforation of the appendix presenting as right thigh abscess. Int Surg. 2002; 87: 61-64.

9. Mukoyama S et al. A Successfully Treated Case of Severe Necrotizing Fasciitis Caused by Acute appendicitis: A Case Report. Tokai J Exp Clin Med 2003; 28(3): 139-43.

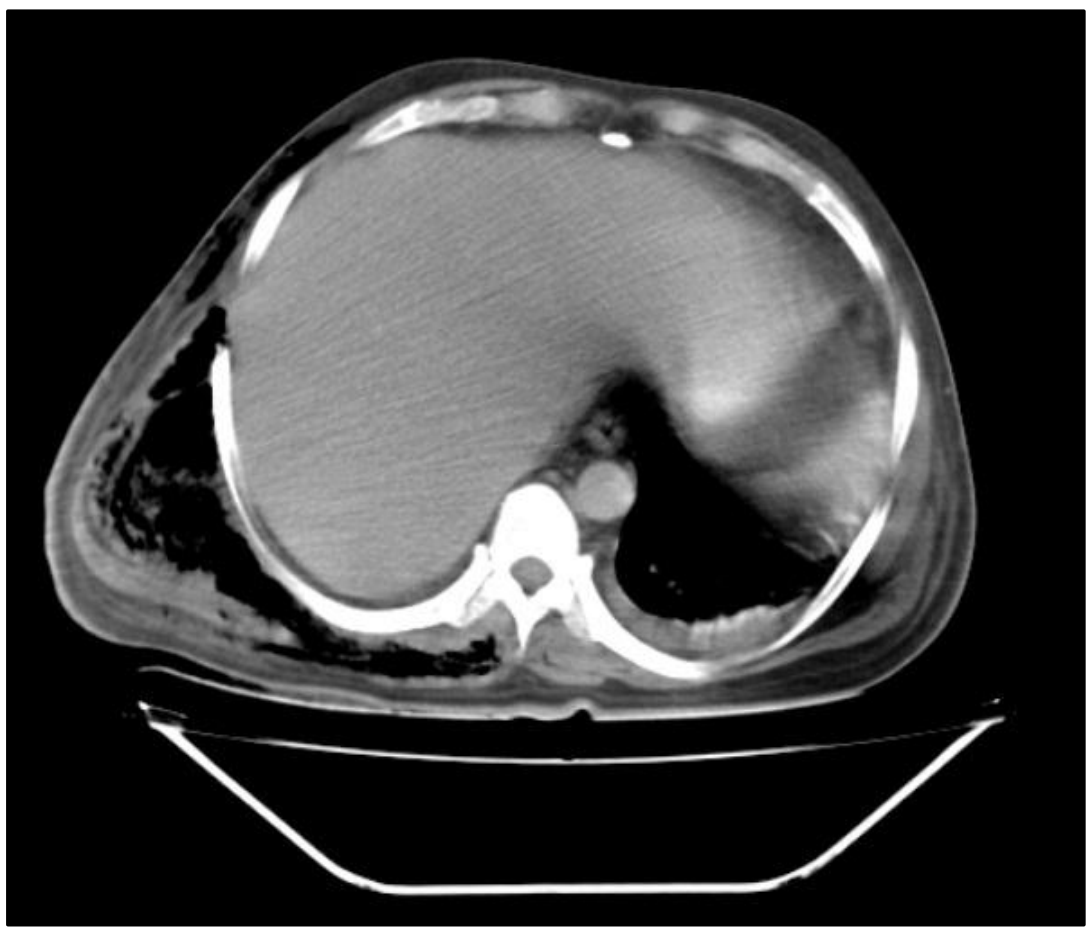

Fig. 1: CT scan of abdomen showing extensive mottled air in the subcutaneous as well as intermuscular plane which is the evidence of necrotizing fasciitis over posterior chest wall \& right flank 


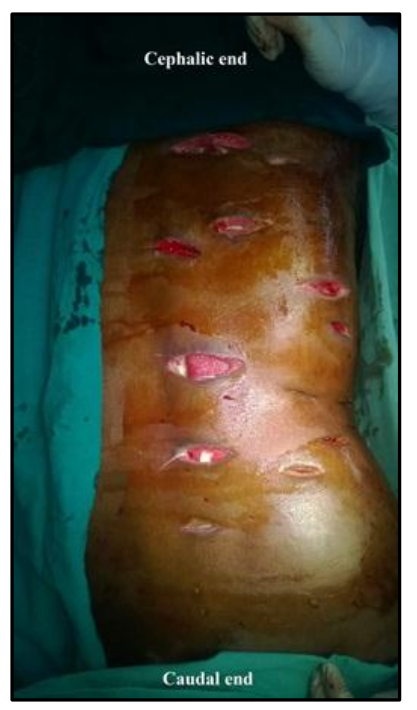

Fig. 2: Showing multiple incisions and drainage with fasciotomy (day 10)

\section{AUTHORS:}

1. Siddharth Mishra

2. Iqbal Ali

3. Gurjit Singh

4. Simran Kaur Bhatia

\section{PARTICULARS OF CONTRIBUTORS:}

1. Junior Resident, Department of Surgery, Dr. D. Y. Patil Medical College, Pimpri, Pune.

2. Professor, Department of Surgery, Dr. D. Y. Patil Medical College, Pimpri, Pune.

FINANCIAL OR OTHER

COMPETING INTERESTS: None

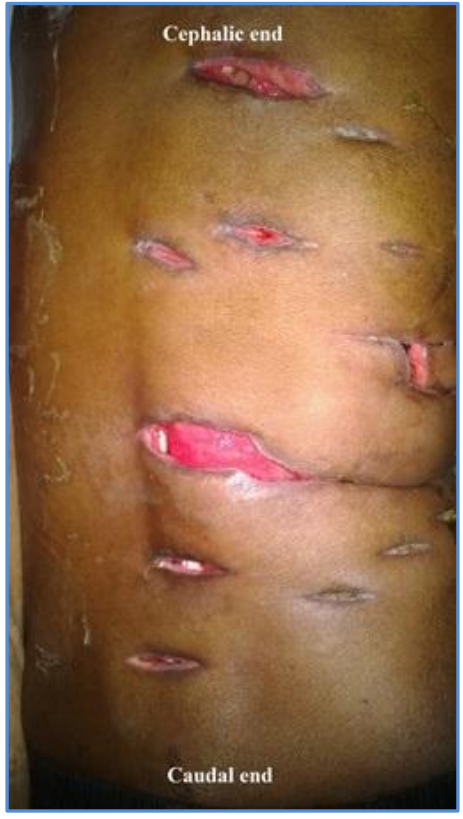

Fig. 3: Incisions were left open and daily Dressing was done (day 20)

3. Professor, Department of Surgery, Dr. D. Y. Patil Medical College, Pimpri, Pune.

4. Intern, Dr. D. Y. Patil Medical College, Pimpri, Pune.

\section{NAME ADDRESS EMAIL ID OF THE CORRESPONDING AUTHOR:}

Dr. Siddharth Mishra,

Department of Surgery,

Dr. D. Y. Patil Medical College Hospital \&

Research Centre, Pimpri, Pune-18.

E-mail: sid1508@gmail.com

Date of Submission: 10/01/2015.

Date of Peer Review: 12/01/2015.

Date of Acceptance: 18/03/2015.

Date of Publishing: 30/03/2015. 